\title{
SÍNTESE E CARACTERIZAÇÃO DE MICROPARTÍCULAS DE AMIDO SOLÚVEL E FÉCULA DE MANDIOCA RETICULADAS COM TRIPOLIFOSFATO DE SÓDIO
}

\author{
E. I. BANDEIRA ${ }^{1 *}$, P. T. MARQUES ${ }^{1}$ \\ ${ }^{1}$ Universidade Tecnológica Federal do Paraná, Departamento de Química \\ *e-mail: estela.bandeira@gmail.com
}

\begin{abstract}
RESUMO
Amido de duas diferentes fontes (solúvel e fécula de mandioca comercial) foram, separadamente, reticulados com tripolifosfato de sódio, em duas diferentes concentrações, $(7,5$ e $15 \% \mathrm{~m} / \mathrm{m})$ a fim de sintetizar micropartículas. Nos espectros de infravermelho das amostras não foi possível visualizar as bandas características das ligações de fosfato ao material formado, porém, desdobramentos na banda característica de hidroxila sugerem modificação na maneira como esse grupo passa a estar ligado. $\mathrm{O}$ conteúdo de amilose nos dois tipos de amido foi mensurado em 21,8\% para o amido solúvel e $28,6 \%$ para a fécula de mandioca. O índice de solubilidade mostra que o processo não modifica a solubilidade do material, porém a adição do agente reticulante aumenta significativamente esse índice, que é de $12,8 \%$ para os controles não processados, 14,7 \% para os controles processados, e de 22,4 \% para a amostra A5R 15 . A densidade de reticulação evidenciou comportamentos diferentes entre os materiais à base de amido solúvel e fécula. Aqueles produzidos com amido solúvel tiveram aumento significativo na densidade de reticulação ao se aumentar a concentração do reticulante, passando de 1,4 para 1,9. Os materiais à base de fécula de mandioca apresentaram comportamento inverso: ao aumentar a concentração do reticulante, a densidade de reticulação diminuiu significativamente de 2,9 para 1,9.
\end{abstract}

\section{INTRODUÇÃO}

Polímeros naturais tem ganhado muita evidência e são objeto de estudos em diversas áreas como a farmacêutica, biomédica, alimentícia, de embalagens, etc. Dentre aqueles que estão sendo mais pesquisados está o amido, que é constituído pelos polissacarídeos amilose e amilopectina. A elevada área superficial e presença de microporos, que os materiais obtidos a partir do amido apresentam, permitem que este possa ser utilizado, por exemplo, como adsorvente na adsorção de metais pesados como cobre (MIAO et al., 2009) e de corantes, como o azul de metileno (GUO et al., 2013).
$\mathrm{O}$ amido mostra-se muito conveniente devido a sua biodegradabilidade, biocompatibilidade, não toxicidade e excelente relação custo-benefício (LI et al., 2012). Sob forma natural apresenta desvantagens, como baixa resistência ao calor e alta suscetibilidade a decomposição, porém através de modificações químicas ou físicas em suas cadeias, um grande leque de alternativas de aplicação passa a existir.

As modificações químicas envolvem a adição de grupos funcionais no amido por reações como eterificação, esterificação, reticulação, enxertia ou decomposição. Um dos métodos químicos mais acessíveis é a reticulação (SOARES et al., 2013). 
O amido pode ser reticulado com diversos agentes reticulantes, dentre os quais os mais utilizados são os que inserem grupos fosfato entre as cadeias e no interior delas, conferindo ao material elaborado caráter aniônico (PARKER e RING, 2001). Um procedimento usual para a estimativa da extensão de reticulação, fundamenta-se nesta característica e nas propriedades do azul de metileno (AM), uma molécula catiônica com grande afinidade por cargas negativas em sólidos (DULONG et al., 2004). Dessa forma, a quantidade de AM adsorvido às micropartículas é proporcional à quantidade de grupos aniônicos inseridos. Quanto maior a quantidade de AM adsorvido, maior a densidade de reticulação promovida, pois o número de grupos fosfato aumenta proporcional à reticulação ocorrida (GAO et al., 2014; LI et al., 2012).

\section{MATERIAL E MÉTODOS}

Nesse estudo, a modificação química foi desenvolvida em duas diferentes fontes de amido: amido solúvel (A.) e fécula de mandioca comercial $(\mathrm{F})$ em meio aquoso. $\mathrm{O}$ reticulante utilizado foi o tripolifosfato de sódio (STPP). O conteúdo de amilose das matérias primas foi caracterizado através de método espectrofotométrico por complexação com iodo. Os efeitos gerados pelo procedimento de reticulação foram avaliados através dos ensaios de estimativa da densidade de reticulação e de índice de solubilidade. A caracterização espectroscópica foi realizada através de Infravermelho com transformada de Fourier.

\subsection{Quantificação do conteúdo de amilose}

O amido solúvel (A) e a fécula de mandioca bruta $(\mathrm{F})$, foram caracterizados quanto a seus conteúdos de amilose através de método espectrofotométrico (MARTINEZ e CUEVAS, 1989), com mudanças na temperatura e tempo de permanência no banho.

\subsection{Síntese de material reticulado}

O material sintetizado foi obtido através do procedimento ilustrado no fluxograma da Figura 1, adaptado de metodologia disponível na literatura (SOARES et al., 2013), com modificação no tempo de reação, proporção de reagentes e natureza do agente reticulante.

Figura 1- Fluxograma do processo de síntese do material reticulado.

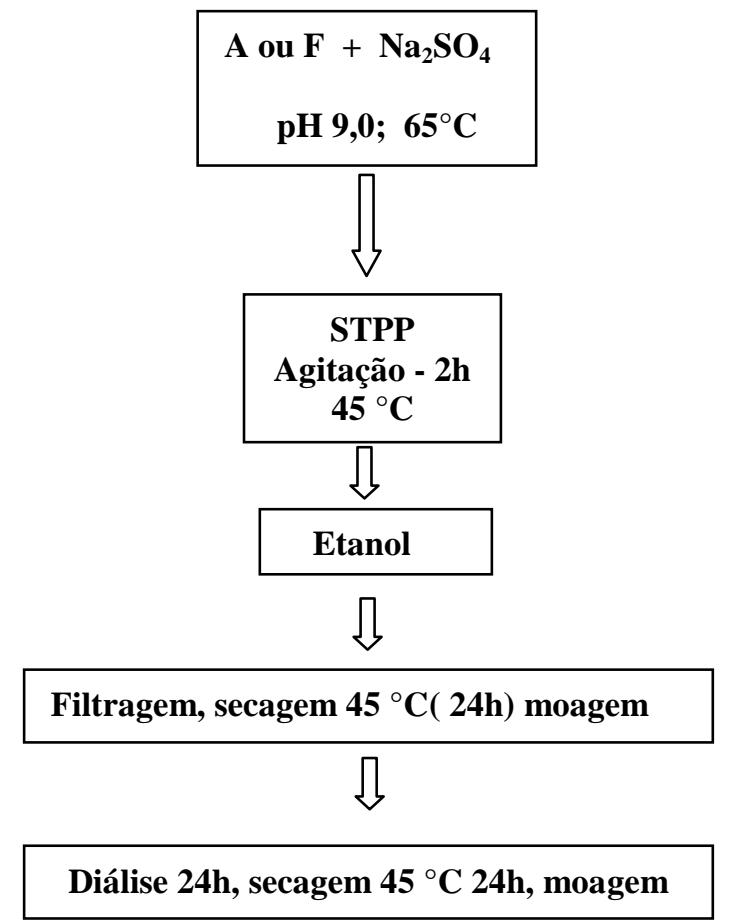

Fonte: adaptado de Soares et al. (2013).

O reticulante STPP foi utilizado em duas diferentes concentrações: $7,5 \%$ e $15 \%$ em relação à massa de amido ou fécula. As amostras foram nomeadas conforme apresentado na Tabela 1. Nesta metodologia utilizou-se dois controles, sendo nomeado processado, aqueles que passaram por aquecimento e $\mathrm{pH}$, sem adição do reticulante (C-A e C-F), e não processado aqueles utilizados como adquiridos, A e F. 
Tabela 1 - Amostras e composição

\begin{tabular}{cc}
\hline Nome & Composição \\
\hline A5R7,5 & $5 \%$ A.; \\
& $7,5 \%$ STPP \\
A5R15 & $5 \%$ A.; \\
& $15 \%$ STPP \\
F5R7,5 & $5 \%$ F.; \\
F5R15 & $7,5 \%$ STPP \\
& $5 \%$ F.; \\
C-A & Controle A. processado \\
C-F & Controle F processado \\
A & Controle A. não \\
& processado \\
F & Controle F. não \\
& processado
\end{tabular}

Fonte: Própria (2015)

\subsection{Estimativa da densidade de reticulação}

A densidade de reticulação (DR) foi mensurada segundo metodologia descrita na literatura (DULONG et al., 2004). As micropartículas reticuladas foram colocadas em contato com uma solução de AM, de concentração e absorbância conhecidas, e o sistema foi armazenado no escuro, por $24 \mathrm{~h}$. Após esse período, foi medida a absorbância residual do AM. A metodologia foi desenvolvida em triplicata. A densidade de reticulação do material foi determinada através da Equação 1:

$$
D R=\frac{C_{O}-C_{1}}{C_{O}-C_{2}}
$$

$\mathrm{Na}$ equação (1) " $\mathrm{C}_{\mathrm{O}}$ " representa a concentração inicial do AM $\left(\mu\right.$ mol.L $\left.L^{-1}\right), C_{1}$, a concentração residual de $\mathrm{AM}\left(\mu \mathrm{mol} . \mathrm{L}^{-1}\right)$ após contato com as amostras reticuladas e $\mathrm{C}_{2}$, a concentração de AM residual, posterior ao contato com os controles $\left(\mu \mathrm{mol} . \mathrm{L}^{-1}\right)$. Denominou-se $\mathrm{DR}_{1}$ o grupo de respostas obtidas para as amostras reticuladas e os controles A e $\mathrm{F}$. $\mathrm{O}$ grupo $\mathrm{DR}_{2}$ é aquele que contém as respostas das amostras reticuladas e dos controles C-A e C-F.

\section{4 Índice de solubilidade (IS)}

$\mathrm{O}$ índice de solubilidade foi mensurado, com adaptações, através de metodologia disponível na literatura (TONGDEESOONTORN et al., 2011). O fluxograma do processo pode ser visualizado na Figura 2.

Figura 2- Fluxograma da metodologia para determinação do índice de solubilidade.

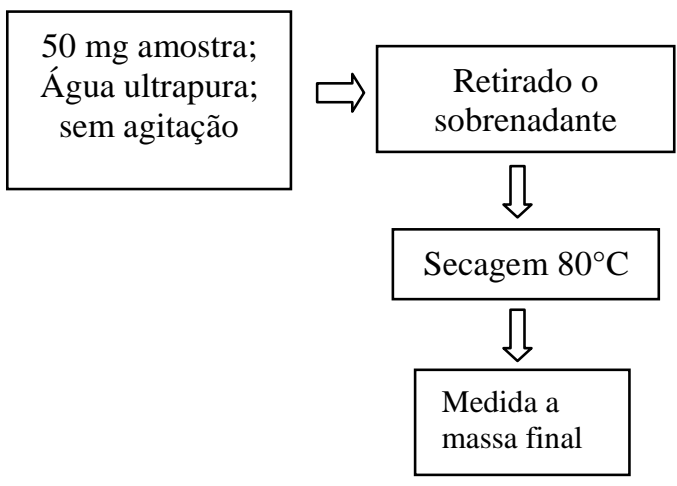

Fonte: adaptado de Tongdeesoontorn et al. (2011).

$O$ índice de solubilização (IS) foi calculado através da Equação 2. Os procedimentos foram realizados em duplicata.

$$
I S=\left(1-\frac{m_{F}-m_{B}}{m_{O}}\right) x 100
$$

$\mathrm{Na}$ equação (2), " $m_{\mathrm{F}}$ " representa a massa final do sistema após secagem $(\mathrm{g}), \mathrm{m}_{\mathrm{B}}$ ", a massa do béquer no início do procedimento $(\mathrm{g})$ e "mo", massa inicial da amostra (g).

\subsection{Caracterização espectroscópica}

Utilizou-se um espectrofotômetro de infravermelho com transformada de Fourier (IVTF), marca Perkin Elmer, modelo Frontier. As amostras previamente secas, moídas em gral e pistilo de ágata e analisadas em pastilhas de $\mathrm{KBr}$.

\section{RESULTADOS}

\subsection{Caracterização espectroscópica}


$\mathrm{Na}$ Figura 3 podem-se observar os espectros de infravermelho dos controles processados (C-A, C-F) e não processados (A, F). Embora o amido solúvel e a fécula de mandioca não sejam de mesma fonte botânica, observa-se que os espectros dos controles não processados são muito semelhantes.

Entre 3200 e $3600 \mathrm{~cm}^{-1}$ uma banda larga aparece devido às vibrações de alongamento do grupo -O-H. Em $2930 \mathrm{~cm}^{-1}$ observa-se uma banda bem definida devido a vibrações de alongamento das ligações -C-H (PENG et al., 2011). É comum observar em espectros de amido, diversas bandas entre 950 e $1200 \mathrm{~cm}^{-1}$, devido a estiramentos da ligação -C-O, com ênfase para as bandas a 1047 e $1022 \mathrm{~cm}^{-1}$, que são sensíveis a alterações na cristalinidade do polímero (SILVERSTEIN, WEBSTER e KIEMLE, 2007). A banda em $1047 \mathrm{~cm}^{-1}$ é associada à porção cristalina, enquanto que a banda em $1022 \mathrm{~cm}^{-1}$ é associada a estrutura amorfa do amido, e sua maior intensidade em relação à banda em $1047 \mathrm{~cm}^{-1}$, evidencia se tratar de um material de característica amorfa (LI et al., 2009).

Figura 3- Espectros de infravermelho normalizados dos controles processados (C-A, CF) e não processados $(\mathrm{A}, \mathrm{F})$

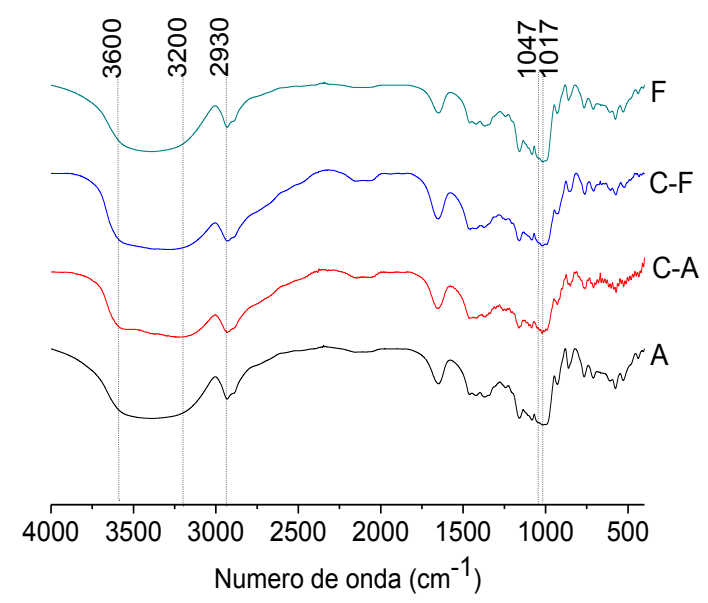

Fonte: Própria (2015)
Ao se comparar os espectros dos controles C-A, C-F e A e F, observa-se que as principais bandas não apresentam alterações significativas, porém a banda característica do grupo -O-H apresenta um leve desdobramento, principalmente para C-A. Sugere-se que esse desdobramento possa ser atribuído ao aumento no número de modos de oscilação, o que pode ser resultado do ambiente alcalino e temperatura do processamento (GARCÍA et al., 2009).

A Figura 4, apresenta os espectros das amostras e do reticulante, STPP. Para este último algumas bandas características sobressaem, como aquelas entre 520 e 665 $\mathrm{cm}^{-1}$, devido ao estiramento assimétrico das ligações $-\mathrm{P}=\mathrm{O}$. Em $895 \mathrm{~cm}^{-1}$ tem-se a banda atribuída ao estiramento vibracional assimétrico do grupo P-O-P, e na região de $1210 \mathrm{~cm}^{-1}$ tem-se bandas atribuídas ao estiramento simétrico de $-\mathrm{P}=0$ (SILVERSTEIN, WEBSTER e KIEMLE, 2007).

Figura 4- Espectro de infravermelho normalizado das amostras e do agente reticulante

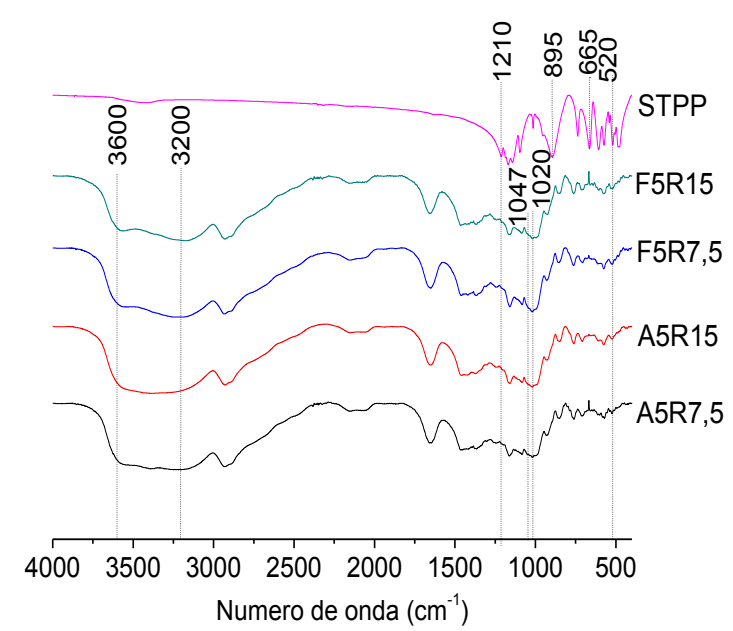

Fonte: Própria (2015)

Ao se observar os espectros percebe-se que, de maneira geral, a banda em $1047 \mathrm{~cm}^{-1}$, pouco intensa nos espectros dos controles, torna-se indiscernível nos espectros das amostras reticuladas. $\mathrm{O}$ aspecto da banda em 
$1022 \mathrm{~cm}^{-1}$, relacionada a mudanças na organização estrutural, mostra que os materiais formados apresentam caráter amorfo, como já citado na literatura (LI et al., 2009).

Bandas características das ligações fosfato $\left(1210 \mathrm{~cm}^{-1}\right)$ que, supostamente acontecem pela inserção do STPP ao polímero, não aparecem nos espectros das amostras reticuladas, porém esse comportamento já foi referenciado na literatura e está associado a baixos níveis de reticulação (LI et al., 2009).

Algumas bandas características do STPP encontram-se na mesma região onde aparecem bandas do amido, o que dificulta afirmar, no caso da banda a $520 \mathrm{~cm}^{-1}$ presente nas amostras reticuladas, tratar-se de uma banda atribuída ao amido, devido a fosfatos adicionados pela reação ou a fosfatos não ligados, residuais na superfície do material.

Observa-se, nos espectros das amostras F5R7,5 e F5R15, que o desdobramento da banda da hidroxila torna-se ainda mais evidente, possivelmente devido à reação de reticulação, onde o STPP liga-se aos $\mathrm{OH}$ do amido (GARCÍA et al., 2009).

\subsection{Quantificação do conteúdo de amilose}

A equação de reta gerada pelas leituras do padrão, $y=0,0221 x-0,0044$, cujo $R^{2}$ foi de 0,9983 , foi utilizada na conversão das absorbâncias, referentes às soluções contendo amido solúvel (A) e fécula de mandioca $(\mathrm{F})$, em concentração de amilose, cujos valores podem ser visualizados na Tabela 2 .

Tabela 2 - Dados de concentração e percentual de amilose obtidos para as amostras de amido solúvel e fécula de mandioca.

\begin{tabular}{ccc}
\hline & $\begin{array}{c}\text { Amido } \\
\text { solúvel }\end{array}$ & Fécula \\
\hline $\begin{array}{c}\text { Concentração } \\
\text { de amilose } \\
\left(\mu \mathrm{g} \cdot \mathrm{mL}^{-1}\right)\end{array}$ & $10,92 \pm 0,19$ & $14,29 \pm 0,03$ \\
$\%$ de amilose & $21,85 \pm 0,38$ & $28,59 \pm 0,06$ \\
Fonte: Própria $(2015)$. &
\end{tabular}

$\mathrm{O}$ percentual de amilose encontrado para A e F, foi $21,85 \% \pm 0,38 \%$ e de $28,59 \%$ $\pm 0,06 \%$, respectivamente. Esses resultados classificam o amido solúvel como sendo de baixo teor de amilose, enquanto que a fécula de mandioca, tem alto teor de amilose (MARTINEZ e CUEVAS, 1989). Sabe-se que a amilose, parte linear do amido, tem grande participação na reação de reticulação, portanto o teor de amilose deve influenciar positivamente a eficiência de reticulação, assim como referenciado na literatura (ZOU et al., 2012).

\subsection{Estimativa da densidade de reticulação}

Como pode ser observado na Figura 5, resultado da aplicação de Teste $\mathrm{T}$ através do programa de livre acesso $R$-comander, há diferenças estatísticas significativas, a nível de $95 \%$ de confiança, entre o grupo de valores $\mathrm{DR}_{1}$ e $\mathrm{DR}_{2}$.

Figura 5 - Comparação entre os grupos de valores de $D_{1}$ e $D_{2}$ através de teste $T$

Comparação entre DR1 e DR2 - Teste T $\square$ Média $\square$ Média \pm Desv pad I Média $\pm 1,96 *$ Desv pad

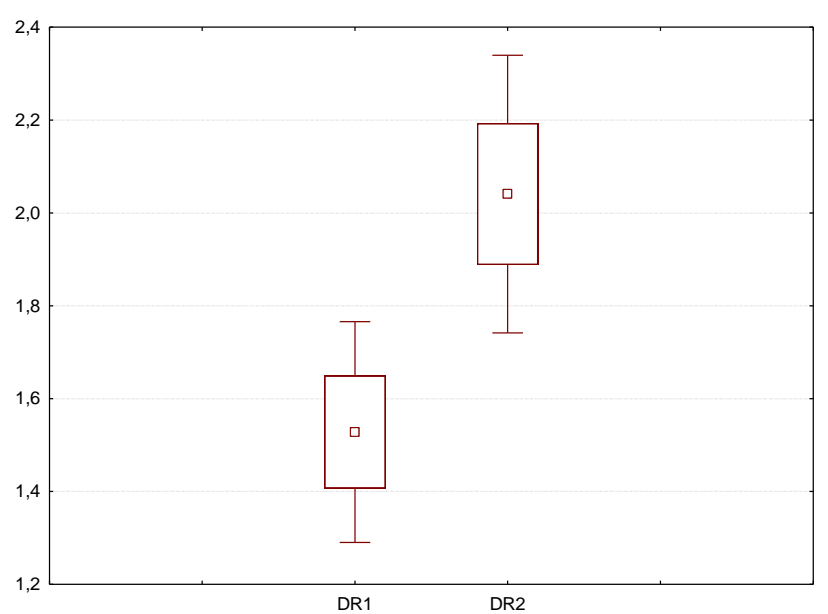

Fonte: Própria (2015)

$\mathrm{DR}_{2}$ foi significativamente superior a $\mathrm{DR}_{1}$, o que evidencia que os controles processados adsorvem menor massa de AM em comparação aos controles não processados. Sugere-se que isso aconteça devido a C-A e C-F terem sofrido pré- 
gelatinização, onde o conteúdo do grânulo de amido é disperso em meio aquoso. Sem o acréscimo do agente reticulante, não há formação de ligações intermoleculares e isso impede o aprisionamento do AM (ONOFRE, WANG e MAUROMOUSTAKOS, 2009).

Ao grupo de dados $\mathrm{DR}_{2}$ foi aplicada análise de variância. Na Figura 6 observa-se que a amostra F5R7,5 apresentou a maior densidade de reticulação sendo estatisticamente diferente das demais, por teste de Fisher, a nível de $95 \%$ de confiança.

Figura 6 - Comparação entre as médias obtidas para cada amostra em $\mathrm{DR}_{2}$. Médias - DRé
Barras verticais denotam intervalo de confiança de 9 $F(3,8)=94,573, p=, 00000$

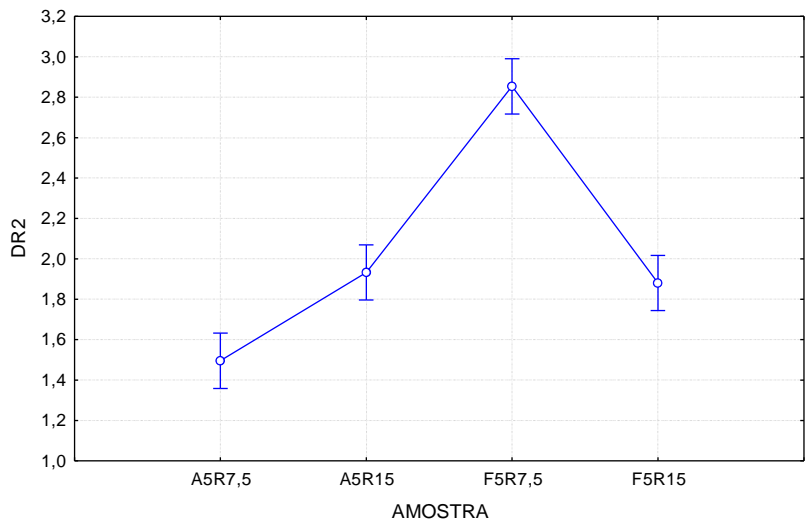

Fonte: Própria (2015)

Já as amostras F5R15 e A5R15 não apresentaram diferenças estatísticas significativas entre si e têm densidade de reticulação intermediária, como pode ser observado na Tabela 3.

Tabela 3 - Comparação entre as médias de densidade de reticulação, através de Teste de Fisher, com 95\% de confiança. Médias com mesma letra em sobrescrito não diferem estatisticamente entre si.

\begin{tabular}{cc}
\hline Amostra & Média DR 2 \\
\hline A5R15 & $1,933^{\mathrm{b}}$ \\
A5R7,5 & $1,496^{\mathrm{c}}$ \\
F5R15 & $1,881^{\mathrm{b}}$ \\
F5R7,5 & $2,854^{\mathrm{a}}$
\end{tabular}

Fonte: Própria (2015)
A amostra que menos reticulou, segundo essa análise foi a A5R7,5 sendo diferente estatisticamente de todas as demais amostras. Essa amostra foi elaborada com amido solúvel que apresenta baixo conteúdo de amilose, sendo portanto que $7,5 \%$ de reticulante pode não ter sido quantidade suficiente para promover uma reticulação extensa. Ao dobrar esse percentual, observase que há um aumento significativo da densidade de reticulação, passando de 1,5 para 1,9, aproximadamente.

No entanto para a fécula, não se observa o mesmo padrão de comportamento. A amostra F5R7,5 apresentou o maior valor de DR, em torno de 2,8, o que pode estar associado ao maior conteúdo de amilose presente na amostra (28\%). Nesse caso, concentração de reticulante superior a 7,5\% apresenta efeito negativo na adsorção do AM e, por consequência, na densidade de reticulação do material. Apesar das amostras terem sido dialisadas, uma das justificativas para esse padrão comportamental diferenciado, pode ser a presença de excesso de sal na superfície desse material, o que poderia estar impedindo a aproximação da molécula de AM.

$\mathrm{Na}$ literatura observa-se uma grande variedade de resultados obtidos em análises que seguem metodologia semelhante, com valores de DR variando entre 0,4 e 46 (LI et al., 2012; DULONG et al., 2004; GLIKOKABIR et al., 2000). O que fica evidente é que o processo de síntese, além da natureza do material, influencia diretamente nos resultados obtidos.

\section{4 Índice de solubilidade}

$\mathrm{Na}$ Figura 7 podem-se observar os IS das amostras, além dos controles processados e não processados. 
Figura 7 - Índices de solubilidade (\%) para cada amostra testada

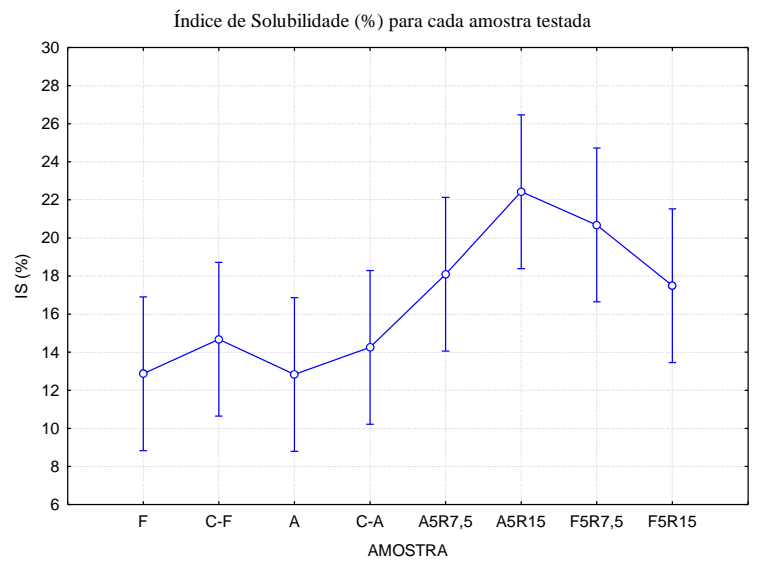

Fonte: Própria (2015)

A esses dados foi aplicada análise de variância e uma comparação entre as médias, utilizando Teste de Fisher, pode ser visualizada na Tabela 4.

Tabela 4 - Comparação entre as médias de índice de solubilidade, através de Teste de Fisher, com 95\% de confiança. Médias com mesma letra em sobrescrito não diferem estatisticamente entre si.

\begin{tabular}{cc}
\hline Amostra & Média IS (\%) \\
\hline A & $12,835^{\mathrm{b}}$ \\
F & $12,869^{\mathrm{b}}$ \\
C-A & $14,259^{\mathrm{b}}$ \\
C-F & $14,682^{\mathrm{b}}$ \\
A5R15 & $22,432^{\mathrm{a}}$ \\
A5R7,5 & $18,093^{\mathrm{ba}}$ \\
F5R15 & $17,497^{\mathrm{ba}}$ \\
F5R7,5 & $20,684^{\mathrm{a}}$
\end{tabular}

Fonte: Própria (2015)

Observa-se que a metodologia de produção das micropartículas não proporciona grandes mudanças no índice de solubilidade dos materiais já que não há diferenças estatísticas significativas, a nível de confiança de $95 \%$, entre C-A, C-F, A e F. Em relação às amostras observa-se que os IS não diferem significativamente entre si, porém os IS das amostras A5R15 e F5R7,5 são significativamente maiores do que seus controles respectivos.
Uma possível explicação para esse aumento na solubilidade pode estar relacionado a baixos níveis de reticulação alcançados através da metodologia seguida, o que facilita a lixiviação de cadeias de amido para a fase aquosa. Um aumento nos níveis de reticulação, impede essa lixiviação, o que resultaria em IS menores (WONGSAGONSUP et al., 2014). Esses mesmos autores obtiveram, para amostras de tapioca reticuladas com trimetafosfato de sódio, IS entre $5,8 \%$ e $2,3 \%$, valores consideravelmente inferiores aos obtidos nesse trabalho.

\section{CONCLUSÃO}

A caracterização das micropartículas sintetizadas neste trabalho possibilitou conhecer o comportamento desse material, imprescindível para avaliar possíveis aplicações. Os espectros de infravermelho, sugerem, pelos desdobramentos observados nas bandas referentes à hidroxila, que a reação de reticulação efetivamente aconteceu, muito embora a banda característica da ligação do grupo fosfato ao material não ser observada. Conclui-se também que o teor de amilose na amostra interfere significativamente e tem efeito positivo no processo de reticulação.

As medidas de índice de solubilidade mostraram que o processo realizado não influencia a solubilidade, porém, a adição do agente reticulante no preparo das amostras aumenta significativamente esse índice.

\section{REFERÊNCIAS}

DULONG, V. ; LACK, S.; CERF, D.; PICTON, L.; VANNIER, J.P.; MULLER, G. Hyaluronan-based hydrogels particles prepared by crosslinking with trisodium trimetaphosphate. Synthesis and characterization. Carbohydrate Polymers, v.57, p.1-6, 2004. 
GAO, F.; LI, D.; BI, C.; MAO, Z.; ADHIKARI, B. Preparation and characterization of starch crosslinked with sodium trimetaphosphate and hydrolyzed by enzymes. Carbohydrate Polymers, v.103, p.310-318, 2014.

GARCÍA, N.L.; RIBBA, L.; DUFRESNE, A.; ARANGUREN, M.I.; GOYANES, S. Physico-Mechanical Properties of Biodegradable Starch Nanocomposites. Macromolecular Materials and Engineering, v.294, p.169-177, 2009.

GUO, L.; LI, G.; LI, J.; MENG, Y.; TANG Y. Adsorptive decolorization of methylene blue by crosslinked porous starch. Carbohydrate Polymers, v.93, p.374-379, 2013.

LI, B.; WANG, L.; LI, D.; CHIU, Y.L.; ZHANG, Z.; SHI, J.; CHEN, X.D.; MAO, Z. Physical properties and loading capacity of starch-based microparticles crosslinked with trisodium trimetaphosphate. Journal lof Food Engineering, v.92, p.255-260, 2009.

LI, B.; WANG, L.; LI, D.; ADHIKARI, B.; $\mathrm{MAO}, \mathrm{Z}$. Preparation and characterization of crosslinked starch microspheres using a twostarge water-in-water emulsion method. Carbohydrate Polymers, v.88, p.912-196, 2012.

MARTINEZ, C. Y.; CUEVAS, F. Evaluación de la calidad culinaria $y$ molinera del arroz. Guia del estudio. Cali: CIAT, 1989.

MIAO, Z.; LI, Z.; DENG, D. WNAG, L. LIU, Y. Novel Crosslinked Starch Microspheres as Adsorbents of $\mathrm{Cu}^{2+}$. Journal of Applied Polymer Science, v.115, p.487-490, 2009.

ONOFRE, $\quad$ F.; $\quad$ WANG, $\quad$ Y.; MAUROMOUSTAKOS, A. Effects of structure and modification on sustained release properties of starches. Carbohydrate Polymers, v.76, p.541-547, 2009.

PARKER, R; RING, S.G. Aspects of the Physical Chemistry of Starch. Journal of Cereal Science, v.34, p.1-17, 2001.

PENG, H.; XIONG, H.; WANG, S.; LI, J.; CHEN, L.; ZHAO, Q. Soluble starch-based biodegradable and microporous microspheres as potential adsorbent for stabilization and controlled release of coix seed oil. European Food Research and Technology, v.232, p.693-702, 2011.

SILVERSTEIN, Robert M.; WEBSTER, Francis X.; KIEMLE, David J. Identificação Espectrométrica de compostos orgânicos. Rio de Janeiro, RJ. LTC: 2007.

SOARES, G.A.; CASTRO, A.D.; CURY, B.S.F; EVANGELISTA, R.C. Blends of cross-linked high amylose starch/pectin loaded with diclofenac. Carbohydrate Polymers, v.91, p.135-142, 2013.

TONGDEESOONTORN, W.; MAUER, L.J.; WONGRUONG, S.; PENSIRI, S.; RACHTANAPUN, P. Effect of carboxymethyl cellulose concentration on physical properties of biodegradable cassava starch-based films. Chemistry Central Journal, v.5, 2011.

ZOU, W.; YU, L.; LIU, X.; CHEN, L. ZHANG, X.; QIAO, D.; ZHANG, R. Effects of amylose/amylopectin ration on starchbased superabsorbent polymers. Carbohydrate Polymers, v.87, p.1583-1588, 2012.

WONGSAGONSUP, R.; PUJCHAKARN, T.; JITRAKBUMRUNG， S.; CHAIWAT，W.; FUONGFUCHAT, A.; VARAVINIT, S.; DANGTIP, S.; SUPHANTHARIKA, M. Effect of cross-linking on physicochemical 
properties of tapioca starch and its application in soup product. Carbohydrate Polymers, v.101, p.656-665, 2014.

\section{AGRADECIMENTOS}

Sinceros agradecimentos à CAPES pela bolsa fornecida e à Central de Análises - UTFPR Campus Pato Branco, pelo uso de suas instalações e equipamentos nas análises de infravermelho. 